\title{
Giant Interatrial Septal Lipoma Filling the Right Atrium Causing Slight Symptoms: A Case Report
}

\author{
Mingxiang Chen, MD,${ }^{1}$ Fuping Li, MD, ${ }^{1}$ Haitao Zhang, MD, ${ }^{2}$ Zhuyun Qin, $\mathrm{MD}^{2}$ \\ ${ }^{1}$ Department of Cardiovascular Surgery, The Third Affiliated Hospital of Chongqing Medical University, Chongqing, 401120, China; \\ ${ }^{2}$ Adult Cardiac Surgical Intensive Care Unit, Fuwai Hospital, Chinese Academy of Medical Sciences and Peking Union Medical \\ College, Beijing, 100037, China
}

\section{ABSTRACT}

Cardiac lipoma is extremely rare. Here, we present a unique illustrative case of an interatrial septal lipoma protruding into the right atrium, causing symptoms in a 54-year-old male. Echocardiogram and computed tomography showed a well-shaped, giant, and fixed mass located in the interatrial septum and right atrium. The only manifestation was palpitation, though the mass filled almost all of the atrium and compressed the superior vena cava. The patient received resection of the large-sized lipoma, which was $87 \mathrm{~mm}$ in diameter and weighed $300-400 \mathrm{~g}$. Pathological exam demonstrated mature lipocytes and substantiated the diagnosis of lipoma. The patient did well postoperatively, and symptoms were resolved.

\section{INTRODUCTION}

Primary cardiac tumor is rare, the incidence is in a range of 1.38 to 30 per 100,000 people per year. Among adult patients over age 16 years old, benign tumors account for $80 \%$ of primary tumors, and $21 \%$ of benign tumors are lipomas [Poterucha 2019]. Patients with cardiac lipoma usually are asymptomatic and can sometimes present with various symptoms, such as heart failure, arrhythmias, cardiac compression, and valvular obstruction. Embolization also can occur if the tumor is not well encapsulated [Rainer 2016]. Due to the low prevalence of cardiac lipoma, there currently are no treatment guidelines. Patients with cardiac lipoma can be clinically observed, and symptomatic patients usually accept tumorectomy. Here, we report the unique case of an illustrative giant cardiac lipoma located in the interatrial septum that protruded into the right atrium, causing symptoms. The patient accepted surgery removal and evaded patching to reconstruct the interatrial septum.

Received fuly 14, 2021; received in revised form September 6, 2021; accepted September 7, 2021.

Correspondence: Zhuyun Qin, MD, Adult Cardiac Surgical Intensive Care Unit, Fuwai Hospital, Chinese Academy of Medical Sciences and Peking Union Medical College, Beijing, China (e-mail: qinzhuyun@163.com).

\section{CASE PRESENTATION}

A 54-year-old East Asian male patient was admitted to the local hospital with a chief complaint of recurrent spontaneous paroxysmal palpitation for one year. This symptom became more frequent for one month. No aberrant signs were found on physical examination. The patient's NT-proBNP was 493.30 $\mathrm{pg} / \mathrm{ml}$ (normal range: $0-125 \mathrm{pg} / \mathrm{ml}$ ). The cardiac function of the patient was class I on NYHA classification. The rhythm was sinus on electrocardiogram (ECG). Echocardiogram presented an ovoid mass located in the right atrium and tightly attached to the interatrial septum. The border of the mass was clear, except the adherent part (Figure 1A, 1B). Left ventricular ejection fraction was $58 \%$. Coronary computed tomography angiography (CTA) revealed a large mass within the interatrial septum and right atrium that took up nearly the entire roof of the right atrium, constricting the superior vena cava (SVC). The upper part of the mass surrounded the aortic root. The size of the mass was $58 \mathrm{~mm} \times 87 \mathrm{~mm} \times 52 \mathrm{~mm}$, and the density was distinctly lower than the peripheral tissues (Figure 1C).

The patient was diagnosed with cardiac lipoma and received surgery through a median sternotomy under cardiopulmonary bypass. An ovoid, yellowish and brownish mass was exposed between the right auricle and SVC after opening the patient's pericardium (Figure 2A). The mass was wellcapsulated, vessel free, and connected with the interatrial septum. Through right atrial atriotomy, a firm, lipoid change of the interatrial septum was presented. Fossa ovalis was absent; tricuspid valve and coronary sinus were not affected. The sessile tumor en bloc was $87 \mathrm{~mm}$ in diameter and gradually isolated by elongating the incision to the roof of the right atrium (Figure 2B, 2C).

Interatrial septum was reconstructed by barely suturing, and there was no incidence that occurred during surgery. Mass sample was performed with hematoxylin-eosin (HE) staining. Adipocytes and myocardial tissue were intersected under a light scope (Figure 3A). The patient did well postoperatively, though there was an intermittent palpitation, the symptom obviously was relieved. Postoperative CT revealed no anomaly (Figure 3B). Frequent ventricular premature beats were found on postoperative ECG when the patient was suffering palpitation (Figure 3C). The patient complained of no other discomfort during the next eight days of in-hospital follow up, and he eventually was discharged. The patient remained asymptomatic on follow up over the next 15 months. 


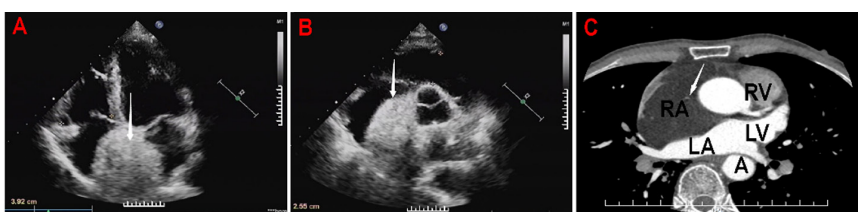

Figure 1. Preoperative ultrasound exam and CT scan. A) Patient's cardiac ultrasonography shows tumor in the interatrial septum (arrow); B) Patient's cardiac ultrasonography shows tumor in the right atrium; C) Preoperative CT scan shows a large mass with low density (arrow) adherent in interatrial septum and filling RA. CT, computed tomography; $\mathrm{RA}$, right atrium; RV, right ventricle; $L A$, left atrium; $L V$, left ventricle; A, aorta
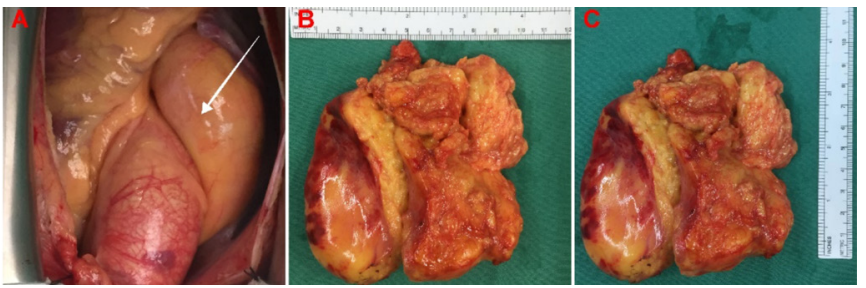

Figure 2. Surgical views of lipoma. A) The view of lipoma (arrow) during operation; B and C) Gross view of resected lipoma specimen.

\section{DISCUSSION AND CONCLUSION}

Cardiac lipomas mostly occur in the age group of 40-60 years [Ismail 2015]. Patients with cardiac lipoma usually are asymptomatic, thus the tumor often is found incidentally during imaging check or autopsy [Bois 2014]. Symptoms caused by cardiac lipoma depends on its size and location. In this reported case, the only clinical manifestation of the patient was palpitation. Adipocyte infiltration in the myocardium attributes to perturbation of myocardial conductivity and leads to arrhythmia [Wong 2017]. Although the preoperative ECG of this patient was normal, postoperative ECG revealed frequent ventricular premature beats, alongside the $\mathrm{HE}$ staining result that mature fat cells and myocardial cells are crossing, indicating that the patient's preoperative palpitation probably was caused by adipocytes causing related arrhythmia. The mechanisms of adipocytes disturb cardiac conductivity is so far poorly elucidated and still in progress of research. Mitochondrial dysfunction, autonomic dysfunction, autophagy, oxidative stress, mitophagy, and myocardial death may all play vital roles in inducing aberrant signals [Pabon 2018].

Cardiac lipoma accounts for $5 \%$ of primary cardiac tumors, it develops from the mesoderm and arises subendocardially, subepicardially, and myocardially [Puvaneswary 2000]. Among these origins, lipomas of subendocardial and subepicardial origin are encapsulated and partially encapsulated as for myocardial origin. Cardiac lipoma can occur anywhere in the heart, of which interatrial septum is the most common position [Monti 2015]. In this case, lipoma at the right atrium was well-enclosed, while in the interatrial septal area, no capsule was presented. It baffled the surgeons, however, whether the origin of the cardiac lipoma in this case arose from the

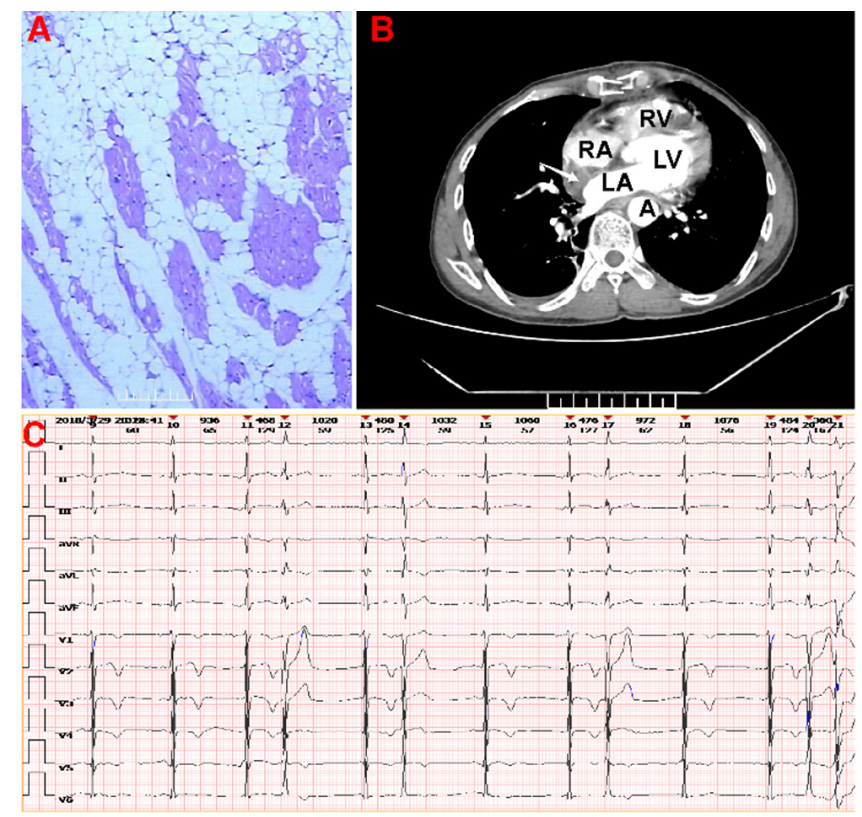

Figure 3. Postoperative pathological findings, CT scan and ECG. A) HE is staining, adipocytes infiltrate into myocardial cells (scale bar: magnification: x100); B) Postoperative CT scan, lipoma has been removed and interatrial septum is reconstructed (arrow); C) Postoperative ECG shows frequent ventricular premature beats. $\mathrm{CT}$, computed tomography; ECG, electrocardiography; HE, hematoxylin and eosin; RA, right atrium; LA, left ventricle; $R V$, right ventricle; $A$, aorta

interatrial septum and protruded to the atrium or arose from the epicardium and infiltrated to the interatrial septum.

Because of the location and the traits of lipoma, interatrial septal lipoma usually is differentiated with lipomatous hypertrophy in interatrial septum (LHIAS). LHIAS and interatrial septal lipoma are both benign lipomatous changes in the heart, consisting of mature fat cells. LHIAS, occurring in $2 \%$ of the population, involves interspersed lipocytes growing within the interatrial septum and making it thicker than $2 \mathrm{~cm}$, and the fossa ovalis is usually spared [Poterucha 2019]. Of note, differing from lipoma, LHIAS is unencapsulated and presents a typical dumbbell shape on CT scan [Hudzik 2010].

Though the etiology of cardiac lipoma is under investigation, genetical change can strongly be related. The rearrangements of two gene members of the high-motility group family, HMGA1 (formerly HMGIY, 6p21) and HMGA2 (formerly HMGI-C, 12q14) have demonstrated involvement in the pathogenesis of $65 \%$ soft tissue lipomas [Rodriguez 2008]. Moreover, amplification of MDM2 and/or CPM, derived from chromosome $12 q 13-15$, also are implicated in lipomatous change [Erickson-Johnson 2009]. Nevertheless, in one study, HMGA2 rearrangement was found in $42 \%$ cardiac lipomas and in 43\% LHIAS cases, while no HMGA1 rearrangement and $\mathrm{MDM} 2 / \mathrm{CPM}$ amplification were demonstrated in either lipomas or LHIAS [Bois 2014]. Therefore, genetic screening can be performed for strengthening the diagnosis and also may be an effective prevention and treatment of cardiac lipoma in the future. 
The scarcity of cardiac lipomas presently brings no treatment guideline. In this reported case, resection was inevitable. SVC was deformed by the tumor, though no symptoms of obstruction were shown, further growth of the mass could have led to potential SVC obstruction syndrome and venal thrombus formation. Even if a lipoma is benign, it can expand in size and oppress the heart, shrinking the volume of the cardiac chambers and impairing heart function, which can be fatal. Furthermore, due to part of this lipoma not being encapsulated, fat liquefaction can occur and subsequently cause fat embolism. The surgical option can decide the outcome of patients with cardiac tumors. Naseerullah et al [Naseerullah 2018] reported a large cardiac lipoma case with surgery removal and reconstruction of the atrial septum and roof of the right atrium using a patch. No patch, however, was used in our case to avoid risk of thrombogenesis and other unpredictable symptoms like arrhythmias. Surgery provides $95 \%$ cure of cardiac benign tumor [Naseerullah 2018], however, surgeons should take the surgery option into consideration so that potential hazards get evitable on patients.

\section{ACKNOWLEDGEMENT}

All authors thank Ruoxi Sun for her assistance on providing information about CT reading. The report was approved by the Ethics Committee of The Third Affiliated Hospital of Chongqing Medical University, and written informed consent was obtained from the individuals for the publication of any images or data included in this article. Clinical trial registration is not applicable for this report.

\section{REFERENCES}

Bois MC, Bois JP, Anavekar NS, Oliveira AM, Maleszewski JJ. 2014. Benign lipomatous masses of the heart: a comprehensive series of 47 cases with cytogenetic evaluation. Hum Pathol. 45:1859-1865.

Erickson-Johnson MR, Seys AR, Roth CW, King AA, Hulshizer RL, Wang X, Asmann YW, Lloyd RV, Jacob EK, Oliveira AM. 2009. Carboxypeptidase M: a biomarker for the discrimination of well-differentiated liposarcoma from lipoma. Mod Pathol. 22:1541-1547.

Hudzik B, Filipiak K, Zembala M, Szkodzinski J, Miszalski-Jamka K, Niklewski T, Glowacki J, Zembala M, Polonski L. 2010. Lipomatous hypertrophy of the interatrial septum: a rare cause of right ventricular impairment. J Card Surg. 25:171-174.

Ismail I, Al-Khafaji K, Mutyala M, Aggarwal S, Cotter W, Hakim H, Khosla S, Arora R. 2015. Cardiac lipoma. J Community Hosp Intern Med Perspect. 5:28449.

Monti L, Scardino C, Nardi B, Balzarini L. 2015. Lipoma of the interventricular septum. Eur Heart J. 36:3073.

Naseerullah FS, Javaiya H, Murthy A. 2018. Cardiac Lipoma: An Uncharacteristically Large Intra-Atrial Mass Causing Symptoms. Case Rep Cardiol. 2018:3531982.

Pabon MA, Manocha K, Cheung JW, Lo JC. 2018. Linking Arrhythmias and Adipocytes: Insights, Mechanisms, and Future Directions. Front Physiol. 9:1752.

Poterucha TJ, Kochav J, O'Connor DS, Rosner GF. 2019. Cardiac Tumors: Clinical Presentation, Diagnosis, and Management. Curr Treat Options Oncol. 20:66.

Puvaneswary M, Edwards JR, Bastian BC, Khatri SK. 2000. Pericardial lipoma: ultrasound, computed tomography and magnetic resonance imaging findings. Australas Radiol. 44:321-324.

Rainer WG, Bailey DJ, Hollis HW, Jr. 2016. Giant Cardiac Lipoma: Refined Hypothesis Proposes Invagination from Extracardiac to Intracardiac Sites. Tex Heart Inst J. 43:461-464.

Rodriguez FJ, Erickson-Johnson MR, Scheithauer BW, Spinner RJ, Oliveira AM. 2008. HMGA2 rearrangements are rare in benign lipomatous lesions of the nervous system. Acta Neuropathol.116:337-338.

Wong CX, Ganesan AN, Selvanayagam JB. 2017. Epicardial fat and atrial fibrillation: current evidence, potential mechanisms, clinical implications, and future directions. Eur Heart J. 38:1294-1302. 